\title{
Word superiority over isolated letters: The neglected case of forward masking
}

\author{
TIMOTHY R. JORDAN and KIM M. BEVAN \\ University of St. Andrews, St. Andrews, Scotland
}

\begin{abstract}
Previous research shows that when briefly presented alphabetic stimuli are followed by pattern masks, letters in words are reported more accurately than are isolated letters (the "WordLetter Phenomenon," or WLP); however, when these masks are replaced by blank fields, the WLP disappears. These findings have led to the popular notion that the WLP reflects selective masking of ongoing stimulus processing and so critically depends on the use of poststimulus masks. Here we report three experiments which re-examine the role of masking in the WLP by contrasting the effects of postmasked displays with the effects of premasked displays in which words and isolated letters were preceded by a pattern mask and followed by a completely blank field. Despite the critical role generally assigned to poststimulus pattern masks, similar WLPs were obtained with pre- and postmasked displays. Implications for theories of word and letter recognition are discussed.
\end{abstract}

By limiting the amount of time for which a word, nonword, or single letter is presented, information can be gained to determine which physical characteristics of words are encoded by the reader and over what time scale this encoding takes place. One particular finding in this area has maintained a considerable impact on contemporary views of word recognition since it was first reported more than twenty years ago. Using a two-alternative forced-choice procedure (generally known as the ReicherWheeler paradigm), a number of studies have shown that when alphabetic stimuli are followed by a pattern mask (composed of irregular arrangements of letter fragments or similar contours), letters in words are reported more accurately than are the same letters presented in isolation (e.g., Carr, Davidson, \& Hawkins, 1978; Carr, Lehmkuhle, Kottas, Astor-Stetson, \& Arnold, 1976; Estes, 1975a; Hawkins, Reicher, Rogers, \& Peterson, 1976; Holender, 1979; Johnston, 1978, 1981; Johnston \& McClelland, 1973, 1974, 1980; Jordan \& de Bruijn, 1993; Massaro \& Klitzke, 1979; Purcell \& Stanovich, 1982; Reicher, 1969; Rumelhart \& McClelland, 1982; Taylor \& Chabot, 1978; Wheeler, 1970). Moreover, this advantage for words appears to be due to the perceptual processing of these items, and not to the use of some form of postperceptual guessing strategy based on orthographic knowledge. For example, when investigating the word advantage over isolated letters, Johnston (1978) argued that

This research was supported by Grant SPG 8931914 from the Joint Council Initiative in Cognitive Science and was reported at the September 1992 meeting of the European Society for Cognitive Psychology in Paris. We are grateful to Hannah Buchanan-Smith, Veronica Dark, Albrecht Inhoff, Geoffrey Loftus, and an anonymous reviewer for help and insightful comments concerning this research. Requests for reprints should be sent to T. R. Jordan, Psychological Laboratory, University of St. Andrews, St. Andrews, Fife KY16 9JU, Scotland. if redundancy were being used at any stage in the processing of word targets, performance with words would be related to the amount by which the identity of the critical letter is constrained by the identities of the remaining letters in each word. Johnston (1978) found no evidence of such a relationship under display and testing conditions similar to those used by Reicher (1969). Thus, when viewing time is limited by the use of a poststimulus pattern mask, letters in words appear to be more perceptible than letters presented in isolation.

Before this advantage for letters in words over isolated letters [the "Word-Letter Phenomenon"' (WLP); Johnston \& McClelland, 1973] can be fully explained, the precise role played by pattern masks in this phenomenon must be revealed (e.g., see Eriksen, 1980). More specifically, while the word advantage over single letters presented in arrays of meaningless characters (e.g., number signs, \#h\#\#; dollar signs, \$h\$\$; ampersands, \&h\&\&) appears to be insensitive to the type of poststimulus field employed (patterned or blank; Johnston \& McClelland, 1973; Marchetti \& Mewhort, 1986; Mewhort \& Johns, 1988), the word advantage over isolated letters (e.g., h ) has so far been observed only when stimuli are followed by pattern masks and disappears when blank poststimulus fields are used (e.g., Johnston \& McClelland, 1973; Juola, Leavitt, \& Choe, 1974; Marchetti \& Mewhort, 1986; Massaro \& Klitzke, 1979; see also Taylor \& Chabot, 1978). For example, Johnston \& McClelland (1973) presented four-letter words (e.g., coin), letters in number signs (c\#\#), and isolated letters (c) in two backwardmasked conditions. In one condition, pattern masks (composed of an irregular pattern of contours resembling letter fragments) were used; in the other condition, each mask was simply a blank white field. Johnston \& McClelland (1973) found that letters in words were reported more accurately than were letters in number signs under both blank- 
field and pattern-masked conditions. However, letters in words were reported more accurately than were isolated letters only when pattern masks were used; when stimuli were followed by a blank field, performance with isolated letters was actually more accurate than it was with words.

The contrasting effects of pattern masks and blank fields on the relative perceptibility of words and isolated letters have inspired the popular notion that the WLP reflects the replacement of ongoing target processing by a subsequently presented pattern mask and, therefore, that the WLP requires the use of poststimulus (hereafter termed backward) pattern masks (e.g., Golden, 1986; Johnston, 1981; Johnston \& McClelland, 1980; McClelland, 1985, 1986, 1991; McClelland \& Rumelhart, 1981, 1988; Paap, Newsome, McDonald, \& Schvaneveldt, 1982; Rumelhart \& McClelland, 1982). For example, consider the accounts of the WLP provided by the interactive-activation model (IAM) of McClelland and Rumelhart (1981; see also McClelland \& Rumelhart, 1988; Rumelhart \& McClelland, 1982) and the hierarchical model (HM) of Johnston (1981). Each model postulates a hierarchical network of detector units forming three levels of representation: one for letter fragments, one for letters, and one for words. Both models contain direct connections between nodes in adjacent levels (i.e., between letter-fragment nodes and letter nodes and between letter nodes and word nodes); however, nodes for letter fragments are not connected directly to nodes for words. When an isolated letter is presented, fragments of the letter are analyzed first, and this information feeds forward into the letter level and activates the appropriate letter node. When a word is presented, similar processes take place at the letter-fragment and letter levels for each letter in the word, but information from activated letter nodes now feeds forward into the word level and activates the appropriate word node. Although the IAM and HM differ somewhat in terms of their functioning (most notably because the HM is a purely feed-forward system, whereas the IAM permits feedback from the word level to the letter level), both models propose that backward pattern masks are necessary for the WLP because the phenomenon itself reflects the selective replacement of ongoing processes of target perception by masking noise. More specifically, when a word or isolated letter is presented, accurate target identification cannot take place until activation in the appropriate representation for the target has built up. This buildup of activation takes time and if, in the meantime, a pattern mask is presented to the system, letter fragments in the mask will add noise to the network and quickly replace the pattern of activation currently existing at the letter level. However, because letter-fragment nodes are not connected directly to word nodes, the pattern of activation currently existing at the word level will be far less susceptible to replacement by masking noise. This disparity in the degree to which ongoing processes of word and letter perception are replaced by the subsequent wave of activation created by a backward pattern mask is, according to these accounts, fundamental to the WLP. In- deed, this interpretation of the influence of backward pattern masks on target perception is also used to explain the absence of the WLP when targets are followed by a blank field because blank fields do not contain the letter fragments necessary to selectively replace the processing of a preceding alphabetic stimulus (see Johnston, 1981; Johnston \& McClelland, 1980; McClelland \& Rumelhart, 1981).

Although the role of masking in the WLP proposed by the IAM and HM may be attractive, two concerns (one empirical, one theoretical) suggest that the importance assigned to backward pattern masks is premature.

First, the empirical concern. Despite the popular interpretation placed on the findings of earlier research into the effects of pattern masks and blank fields on word-letter performance, this research actually provides only equivocal support for the notion that backward pattern masks are a necessary component of the WLP. Specifically, while Johnston and McClelland (1973), Juola et al. (1974), Marchetti and Mewhort (1986), Massaro and Klitzke (1979), and Taylor and Chabot (1978) found that the WLP disappeared when blank fields were used instead of backward pattern masks, these findings were actually obtained using displays in which a blank field both followed and preceded each stimulus. Specifically, while each study used blank poststimulus fields, Juola et al. (1974), Marchetti and Mewhort (1986), Massaro and Klitzke (1979), and Taylor and Chabot (1978) preceded each word and isolated letter with a plain dark field, and Johnston and McClelland (1973) used a plain white field for both poststimulus mask and prestimulus fixation field. Therefore, rather than demonstrating the critical importance of backward pattern masks in the WLP, these studies actually show that the WLP disappears when pattern masks are absent from each trial entirely, not just from the poststimulus field. Consequently, rather than indicating that backward pattern masks are necessary for the WLP, such studies demonstrate that the WLP may require merely that a pattern mask be presented on each trial, irrespective of whether this is before or after each word or isolated letter is shown. ${ }^{1}$ Thus, a more informative way to investigate the role of backward pattern masks in the WLP is to contrast performance under backward-masked conditions (where words and isolated letters are preceded by a blank field and followed by a pattern mask) with performance under forward-masked conditions (where words and isolated letters are preceded by a pattern mask and followed by a blank field). In this way, not only would backward pattern masks be replaced by a blank poststimulus field (the critical comparison for backward-masking theorists), but the overall content of forward patternmasked displays would also match that of backward patternmasked displays, where the WLP has traditionally been observed. In so doing, problems of interpretation associated with contrasting backward-masked performances with those obtained using "control" displays in which stimuli are presented without any pattern mask at all would be overcome. 
We turn now to the second, theoretical, concern. Interruption masking is not the only prevailing theory that can explain why pattern masks disrupt the perception of alphabetic targets. For example, it has been known for some time that the sensory qualities of target and mask can become integrated to form a composite percept incorporating aspects of both stimulus fields (e.g., see Breitmeyer, 1984; Felsten \& Wasserman, 1980; Ganz, 1975; Jordan, 1990, in press). Thus, although backward pattern masks have the potential for interrupting target processing, it is far from clear that interruption, rather than integration, plays a critical role in the WLP. Indeed, Jordan and de Bruijn (1993) have recently argued that a possible role for backward pattern masks in the WLP is that of selectively impairing the discriminability of isolated letter targets through target-mask integration. More specifically, when words and isolated letters are presented under backward pattern-masked conditions, both types of target may become formed into composite percepts of target and mask. However, isolated letters (because of their diminutive size) may be less easily discriminated than words from each pattern mask, which may inspire an advantage for word targets (see also Estes, 1975a, 1975b). This account can also explain the absence of the WLP when blank fields are used. Quite simply, the word advantage over isolated letters disappears when blank fields are used because blank fields do not contain the contours necessary to selectively impair target discrimination. Thus, when pattern masks are used, the structural similarity between the contours of each target and mask may disguise the precise location and extent of each target, but the absence of these contours when blank fields are used allows both words and isolated letters to be readily discriminated in each display. Consequently, while blank fields may impair performance in other ways (e.g., by lowering target contrast when a blank white flash is used, as Johnston \& McClelland, 1973, suggest), the discriminability of isolated letters may not be selectively impaired in these displays, and so the possibility of obtaining a word advantage is removed.

The accuracy of Jordan and de Bruijn's (1993) integration-discrimination hypothesis has yet to be fully determined. At the very least, however, the hypothesis proposes that the role of masking in the WLP is not interruptive and that the WLP is produced because words and isolated letters differ with respect to the ease with which they can be discriminated from the masking stimulus. If this were the case, there seems little reason to suppose that a WLP should not also be produced under forwardmasked conditions; a comprehensive examination of the literature reveals that this critical test of the role of masking in the WLP has never been reported.

The picture being developed, therefore, is of a WLP that may be inspired under both backward- and forwardmasked conditions. But, as we have already shown, this view contrasts sharply with current accounts of the role of masking in the WLP. Indeed, for the HM, a word advantage "depends on the use of a patterned mask following target exposure"' (Johnston, 1981, p. 76) while, for the IAM, pattern masks inspire a word advantage by "driving the system towards a new steady state and wiping out the remaining traces of the previous stimulus", (Rumelhart \& McClelland, 1982, p. 61). However, while the accuracy of these accounts has not yet been challenged by the report of a WLP under forward-masked conditions, the role of masking that they propose has already been challenged by a recent study by Prinzmetal (1992). Prinzmetal was also concerned with the notion that backward pattern masks are necessary for the WLP, and examined its accuracy by presenting a pattern mask simultaneously with each stimulus. Despite the fact that no backward mask was presented on any trial, an advantage for words over isolated letters was observed (Prinzmetal, 1992, Experiments 4 and 5). However, Prinzmetal was concerned with establishing a WLP without brief presentations and so limited performance by using unusually small stimuli (for example, four-letter words subtended only $0.426^{\circ}$ ) which were presented until subjects responded. This deviation from the conditions under which the WLP has traditionally been reported makes it difficult to compare Prinzmetal's findings with those of previous research in this area. Moreover, Prinzmetal did not employ a backward-masked condition in his experiments and so provided no benchmark with which to compare the WLP obtained without backward masks. This raises the possibility that a stronger word advantage might be obtained when backward pattern masks are used and, therefore, that backward pattern masks still play a special role in the WLP. Therefore, while the findings of Prinzmetal offer encouragement for the view that backward pattern masks are not necessary for the WLP, the precise extent to which backward pattern masks inspire the WLP traditionally observed under abbreviated viewing conditions remains unclear. From the arguments presented earlier, a comparison of the effects of forward and backward pattern masks on the relative perceptibility of briefly presented, "normal-sized" words and isolated letters is needed to resolve the role of masking in the WLP.

In sum, therefore, the three experiments reported in this article were motivated by the view that the WLP traditionally observed with backward pattern masks may also be observed with forward pattern masks even though, under forward-masked conditions, words and isolated letters are followed by a completely blank field. Moreover, by directly comparing the effects of forward and backward pattern masks on the relative perceptibility of words and isolated letters, information will be gained about the precise involvement of pattern masks in the WLP; if similar word advantages are observed with backward- and forward-masked displays, the popular notion that backward pattern masks play a special role in the WLP (e.g., as proposed by the IAM and HM) will require substantial revision.

\section{EXPERIMENT 1}

Experiment 1 was conducted as a straightforward test of the hypothesis that the WLP can be produced even 
when words and isolated letters are preceded by a pattern mask and followed by a completely blank field.

\section{Method}

Subjects. Sixteen paid subjects participated in the experiment. All subjects had normal or corrected-to-normal vision and were native speakers of English.

Stimuli. Ninety-six pairs of four-letter words were selected as experimental stimuli, with a mean frequency of written occurrence of 123 per million (Carroll, Davies, \& Richman, 1971). The members of each pair differed by just one "critical" letter (e.g., show, snow), with critical letters occurring equally often at each of the four letter positions. Target stimuli were constructed from a proportionally spaced character set which provided natural variation in the width of words and letters. This variation avoided presenting the individual letters of target stimuli in just four absolute screen positions, since this may have provided abnormal cues to the positions of letters in targets; in addition, the two critical letters of each stimulus pair shared the same width and spacing to avoid response strategies based on the width of each display. Ninety-six pairs of isolated-letter stimuli were formed by deleting the three noncritical letters from each word pair, leaving each isolated letter in exactly the same screen position as it appeared in the word. Two groups of 96 stimuli were formed from these word and isolated-letter stimuli, with each group containing one member of each word pair and its matched isolated letter. An additional 40 word pairs and 40 letter pairs were constructed to provide 80 practice stimuli at the beginning of each session.

For each trial, a different pattern mask was constructed from pseudorandomly arranged fragments of the letters used in the character set, with the built-in constraint that no letters were formed by these fragments. A preliminary detection task showed that these masks greatly impaired perceptibility relative to a no-mask condition, and rendered words and isolated letters undetectable when stimulus exposures were sufficiently brief. The character set and example mask and word stimuli are shown in Figure 1.

Visual conditions. Masks, words, and isolated letters were presented in white on a dark gray oscilloscope screen. Words and letters were presented in a proportionally spaced, lowercase font, based on the Letraset "Pin Ball" typeface (see Figure 1). Background illumination of the oscilloscope screen was approximately $1 \mathrm{~cd} / \mathrm{m}^{2}$, and the luminance of stimuli and masks was approximately $25 \mathrm{~cd} / \mathrm{m}^{2}$.

A single letter $x$ subtended horizontal and vertical visual angles of approximately $0.20^{\circ}$. Four proportionally spaced letter $x \mathrm{~s} \mathrm{sub-}$ tended a visual angle of approximately $1.10^{\circ}$ horizontally. Same-

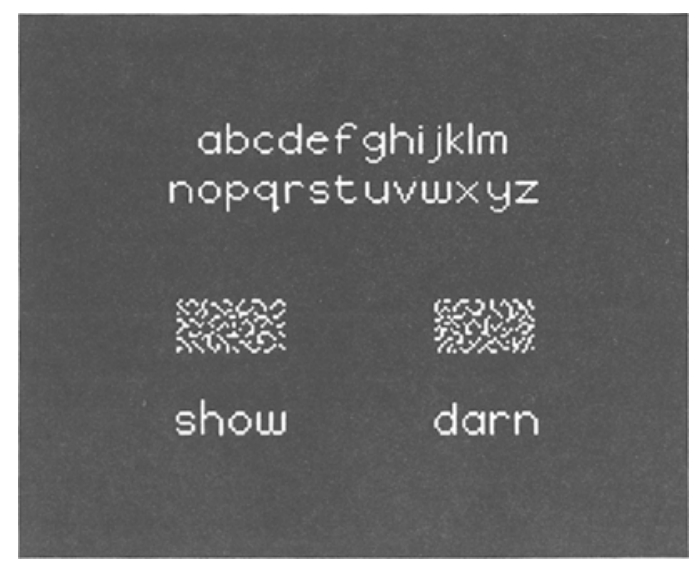

Figure 1. The character set and example mask and word stimuli used in Experiment 1. sized masks were used for words and isolated letters. Each mask subtended a visual angle of approximately $0.50^{\circ}$ vertically and a horizontal visual angle individually matched to the width of each word target.

Design. Subjects took part in two sessions, one on each of 2 different days. Half of the subjects were shown Stimulus Group 1 and half were shown Stimulus Group 2; for each subject, the same stimulus group was shown in both sessions. Each session was divided into three sections (practice, A, and B), with no obvious transition from one section to the next. In each session, each word and yoked isolated-letter stimulus were presented in a different section of the experiment (A or B); this ensured that the members of each word/ isolated-letter pair were not presented in close succession. The allocation of stimuli to sections A and B was re-randomized for each subject, with the constraint that each section contained an equal number of each stimulus type ( 48 words and 48 isolated letters). Stimuli were shown in cycles of 16 trials, counterbalanced across stimulus type and critical-letter position.

Apparatus. The experiment was controlled by a Cambridge Electronic Design 1401 intelligent interface slaved to a computer. Stimuli were plotted on a Hewlett-Packard 1332A oscilloscope equipped with rapid-decay $\mathrm{P} 4$ phosphor with a spot persistence time of 10 $\mu \mathrm{sec}$ to $10 \%$. The screen of the oscilloscope was completely covered with matte black card except for an area at its center measuring approximately $1^{\circ}$ vertically and $2^{\circ}$ horizontally. In addition, the oscilloscope had been modified to enable precise control over the visual angle of stimuli and to provide a higher resolution display (Jordan \& Martin, 1987). The experiment was conducted in a darkened booth, and the subjects entered their responses via two illuminated keys interfaced with the computer.

Procedure. At the beginning of their first session, each subject was familiarized with all 26 letters of the character set used in the experiment. At the start of each trial, a small fixation point appeared at the center of the screen. The subjects were instructed to look at this point when initiating a display. When they pressed a key, the fixation point disappeared and the following display sequence was initiated: 500 msec blank; mask; stimulus; 500 msec blank. Exposure durations for stimuli were determined individually for each subject (see below); masks were presented for $50 \mathrm{msec}$ longer than was each stimulus as an aid to the effectiveness of masking. Four dashes were then shown, corresponding to the four letter positions in a four-letter word. At one of these dashes, two letters were shown, one above the dash and one below, and the subjects had to decide which of these two letters had been shown at the position indicated by the dash. To make their choice, the subjects pressed one of two keys to select either the upper or the lower alternative. For isolated letters, the dashes were somewhat redundant, but the same procedure was used for both types of stimulus.

Throughout the practice and experimental sections, exposure durations were reassessed for each subject after each cycle of 16 trials. Exposure duration was increased (by $2 \mathrm{msec}$ ) if the number of correct responses in a cycle was below $11(68.75 \%)$ and was decreased (by $2 \mathrm{msec}$ ) if the number of correct responses in a cycle was above $13(81.25 \%)$. Within each cycle, words and isolated letters were shown for the same exposure duration; when adjustments to exposure duration were made at the end of a cycle, the same adjustment was made for both types of target. This adjustment procedure ensured that overall performance fell in the midrange of the performance scale and that each condition was represented at the same exposure duration an equal number of times. Average exposure duration for words and isolated letters was $48 \mathrm{msec}$.

\section{Results and Discussion}

The raw data were submitted to an analysis of variance (ANOVA) with one between-subjects factor (stimulus group) and one within-subjects factor (stimulus type). The findings were clear: mean correct performance was $73.2 \%$ 
for words and $65.1 \%$ for isolated letters; the $8.1 \%(S E=$ $\pm 1.34 \%)$ advantage for words was significant $[F(1,14)=$ $\left.7.74, M S_{\mathrm{e}}=13.24, p<.01\right]$ and showed no sign of interacting with stimulus group $\left[F(1,14)=.039, M S_{\mathrm{e}}=\right.$ $6.48, p>.80]$. Thus, the WLP does not require the use of backward pattern masks even when stimuli are briefly presented and of a normal size (cf. Prinzmetal, 1992) and may be observed even when targets are preceded by a pattern mask and followed by a completely blank field (cf. the IAM and HM).

\section{EXPERIMENT 2}

Experiment 1 established the existence of a WLP under forward pattern-masked conditions; however, this finding is novel and requires replication. In addition, the extent to which the WLP may differ under forward and backward pattern-masked conditions has yet to be determined. Therefore, Experiment 2 was conducted to provide a direct comparison between the size of the WLP observed with forward and backward pattern-masked displays.

Previous research suggests that pattern masks are generally more disruptive in forward-masked displays (e.g., Kietzman, Boyle, \& Lindsley, 1971; Michaels \& Turvey, 1979; Schiller, 1966; Schiller \& Smith, 1965; Smith \& Schiller, 1966; Sperling, 1960, 1965; Turvey, 1973); a preliminary investigation of the forward- and backwardmasked displays planned for Experiment 2 revealed a similar difference between masking conditions. Specifically, when the same exposure durations were used for words and isolated letters in forward- and backward-masked displays, overall performance with forward masks was barely better than chance $(55 \%)$, while performance with backward masks came close to perfection (96\%). Thus, in order to obtain an appropriate comparison between the effects of forward and backward pattern masks on the size of the WLP, overall levels of performance for the forward- and backward-masked displays used in Experiment 2 were matched by adjusting exposure durations separately for each mask condition.

\section{Method}

Subjects. Twenty-four subjects from the same population as that used in Experiment 1 participated in Experiment 2.

Stimuli. The stimuli used in Experiment 1 were used in Experiment 2. Pattern masks were constructed as before, but were now used in two mask conditions. Forward-masked displays were identical to those of Experiment 1, comprising the sequence: fixation point; 500 msec blank; mask; stimulus; 500 msec blank. Backwardmasked displays comprised the same components, but in the following sequence: fixation point; 500 msec blank; stimulus; mask; $500 \mathrm{msec}$ blank. All masks were presented for $100 \mathrm{msec}$.

Design. As in Experiment 1, subjects took part in two sessions, one on each of 2 different days. In Session 1, half the words and their matched isolated letters were shown in the forward-masked condition and half in the backward-masked condition. In Session 2, masking conditions were reversed. Stimuli were shown in cycles of 16 trials, counterbalanced across mask type (forward, backward), stimulus type, and critical-letter position.

Exposure durations were reassessed individually for forward- and backward-masked displays and adjusted if performance differed sub- stantially from $75 \%$ correct. Reassessment occurred after every 16 trials for practice stimuli and after every 32 trials for experimental stimuli. All remaining aspects of this experiment were the same as for Experiment 1.

\section{Results}

Two types of data were collected in this experiment, namely, accuracy of report and stimulus exposure durations. These two data sets were each subjected to an ANOVA with one between-subjects factor (stimulus group) and two within-subjects factors [mask type (forward, backward) and stimulus type (word, isolated letter)]. The individual assessment of performance for each mask condition produced the desired effect; similar levels of overall accuracy were obtained with forward and backward masks $[69.2 \%$ for forward-masked displays, $69.6 \%$ for backward-masked displays; $F(1,22)=2.06, M S_{\mathrm{e}}=$ $11.16, p>.10]$. This matching was achieved by presenting stimuli in forward-masked displays for a mean of $47.25 \mathrm{msec}$, compared with a mean of $12.48 \mathrm{msec}$ for stimuli in backward-masked displays $[F(1,23)=1190.57$, $\left.M S_{\mathrm{e}}=1.35, p<.0001\right]$.

Accuracy data for Experiment 2 are shown in Figure 2. Overall performance for words was $8.8 \%$ more accurate $(S E= \pm 0.66)$ than it was for isolated letters $[F(1,22)=$ $\left.92.37, M S_{\mathrm{e}}=3.26, p<.0001\right]$. No other main effects or interactions were found. The advantage for words across forward-and backward-masked conditions was examined more closely by a subsidiary ANOVA for each mask condition. A WLP was observed with each type of mask: for forward-masked displays, the word advantage was $8.3 \%\left[S E= \pm 0.38, F(1,23)=19.11, M S_{\mathrm{e}}=4.12\right.$, $p<.001]$; for backward-masked displays, the word advantage was $9.3 \%[S E= \pm 0.73, F(1,23)=61.61$, $\left.M S_{\mathrm{e}}=3.91, p<.001\right]$. Neither WLP showed any sign of interacting with stimulus group (both $F \mathrm{~s}<1$ ).

\section{Discussion}

The findings of Experiment 2 add further support to the view that the WLP does not require the presentation of a backward pattern mask. Moreover, the word advantage observed with backward masks decreased only slightly (by $1 \%$ ) when forward masks were used, suggesting that much

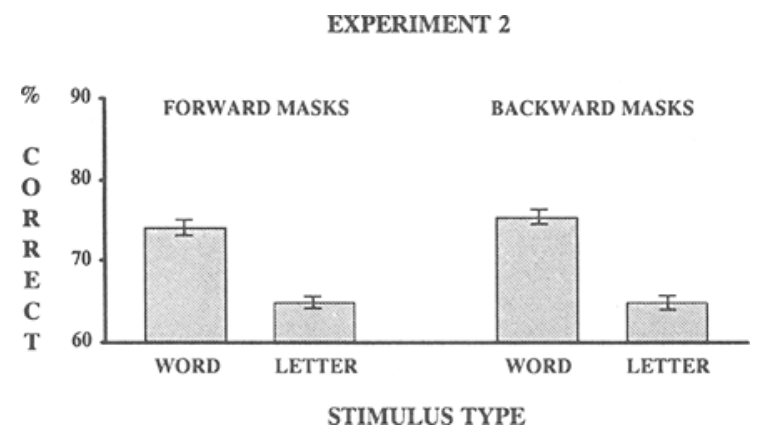

Figure 2. Mean percentage of critical letters correctly reported for words and isolated letters in the forward-and backward-masked displays of Experiment 2. Error bars represent standard errors. 
of the influence of backward pattern masks on the WLP remained under forward-masked conditions. This similarity between forward- and backward-masked displays underscores the problems associated with contemporary accounts of the WLP in which special powers are attributed to backward pattern masks; from the findings of Experiment 2 , the word advantage over isolated letters shows little sensitivity to the temporal ordering of stimulus and mask.

While a similar-sized WLP was observed with forwardand backward-masked displays in Experiment 2, overall levels of accuracy were equated by presenting stimuli for longer durations in forward-masked displays, which suggests that pattern masks were generally more disruptive when presented before a stimulus was shown. As discussed earlier, this finding is consistent with previous comparisons of the effects of forward and backward pattern masks (e.g., Kietzman et al., 1971; Michaels \& Turvey, 1979; Schiller, 1966; Schiller \& Smith, 1965; Smith \& Schiller, 1966; Sperling, 1960, 1965; Turvey, 1973), which indicates that the different degrees of masking observed in Experiment 2 were not a quirk of that particular experiment. Moreover, as contemporary accounts of the WLP assign no special importance to particular exposure durations in backward-masked displays, there is no principled reason why the longer exposure durations necessitated by the use of forward pattern masks should detract from the finding that similar forms of the WLP were obtained under backward- and forward-masked conditions. Nevertheless, for the purposes of comparing the two forms of the WLP, greater reliance could be placed on their apparent similarity by knowing more precisely how exposure duration relates to the relative perceptibility of words and isolated letters in each mask condition. For example, longer exposure durations with forward pattern masks suggest that stimulus perception (words and letters) in forward-masked displays occurred over a longer time scale than it did in backward-masked displays. Consequently, and importantly for comparisons between the two forms of WLP, these different time scales may reflect different temporal relationships between word and letter perception for each type of mask; that is, the perception of words and isolated letters under forward-masked conditions may have been separated by a greater delay than under backward-masked conditions, even though similar overt word advantages were observed with each mask condition in Experiment 2. On the other hand, a great deal of evidence indicates that masking can occur at different stages of perceptual processing: peripherally (occurring from the retina up to, and perhaps including, primary projection areas of the visual cortex) and centrally, where the output of peripheral analyzers is integrated into relational or categorical features by cortical analysis (for reviews, see Breitmeyer, 1984; Breitmeyer \& Ganz, 1976; Kahneman, 1968; Kolers, 1968; Turvey, 1973). In addition, many researchers argue that forward masks create much of their interference at a peripheral stage of per- ception and that peripheral masking is greater with forward masks than it is with backward masks (e.g., Greenspon \& Eriksen, 1968; Kietzman et al., 1971; Michaels \& Turvey, 1979; Moscovitch, 1986; Schiller, 1966; Schiller \& Smith, 1965; Smith \& Schiller, 1966; Sperling, 1960, 1965; Turvey, 1973). Applying this logic to the findings of Experiment 2, the longer exposure durations required for forward masks may reflect merely an overall disadvantage for forward-masked stimuli at a peripheral stage, outside the word-letter-recognition module (e.g., outside the modules represented by the IAM and $\mathrm{HM}$ ), and word perception and letter perception may be separated by a similar temporal delay under forwardand backward-masked conditions. Thus, while forward and backward pattern masks in Experiment 2 produced a similar WLP, it remained to be seen whether these overt word advantages were produced by the same dynamics of processing in each mask condition (see McClelland, 1991, for further discussion of the importance of establishing the temporary dynamics of processing). ${ }^{2}$

One way to examine the dynamics of processing underlying the WLP in forward- and backward-masked displays is to establish the amount of increase in exposure duration for isolated letters that is required to remove the word advantage in each mask condition. ${ }^{3}$ More specifically, if the word advantages observed with forward and backward masks in Experiment 2 were each produced by the same temporal difference between perceiving letters in words and letters in isolation, the word advantage should be removed in each mask condition by a similar increase in exposure duration for isolated letters. If, however, the longer exposure durations used with forward masks concealed a greater temporal difference between perceiving letters in words and letters in isolation, a greater increase in exposure duration for isolated letters should be required to remove the word advantage from forward-masked displays. If different increases in exposure duration are necessary, it will be difficult to argue that the same processing dynamics are responsible for both forward- and backwardmasked forms of the WLP.

\section{EXPERIMENT 3}

The procedure used in Experiment 3 was adapted from Turvey (1973; Experiment 10) to make a procedure that was more applicable to the Reicher-Wheeler paradigm. Specifically, exposure durations were adjusted independently for words and isolated letters in forward- and backward-masked displays so that forced-choice performance was aimed at $75 \%$ correct for each stimulus condition. In this way, the relative perceptibility of each stimulus type (word, isolated letter) $\times$ mask type (forward, backward) combination was revealed by differences in exposure duration, using testing conditions and a level of performance matched to those employed when the WLP has previously been observed. 


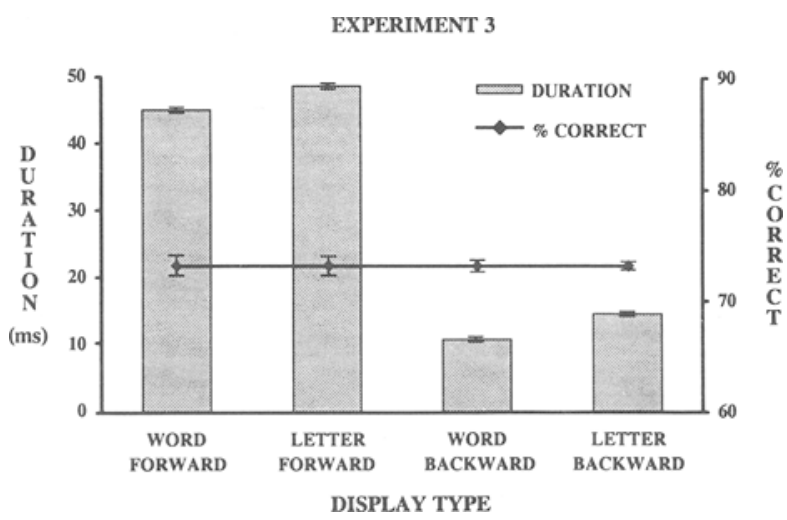

Figure 3. Mean exposure durations (in msec) for words and isolated letters in the forward- and backward-masked displays of Experiment 3. The line data refer to the mean percentage of critical letters correctly reported in each condition. Error bars represent standard errors.

\section{Method}

Subjects. Sixteen paid subjects from the same population as that used in Experiments 1 and 2 participated in the experiment.

Design. Throughout the practice and experimental sections, stimuli were shown in cycles of 16 items, representing the 8 stimulus type $\times$ critical-letter position combinations for each mask condition. Within each cycle, stimuli were shown in a random order, and exposure durations for words and isolated letters in each mask condition were independently assessed after all 8 items for that mask condition had been shown. All remaining aspects of this experiment were the same as for Experiment 2.

\section{Results}

The results of Experiment 3 are shown in Figure 3. As in Experiment 2, two types of data were collected: accuracy of report and stimulus exposure durations. These two data sets were each subjected to an ANOVA with one between-subjects factor (stimulus group) and two withinsubjects factors [mask type (forward, backward) and stimulus type (word, isolated letter)]. Turning first to the data for accuracy of report, no main effects or interactions were obtained (all $p s>.15$ ). Indeed, virtually identical levels of performance were obtained for words and isolated letters in forward- and backward-masked displays: for forward masks, $73.1 \%$ (words) and $72.2 \%$ (isolated letters); for backward masks, $73.2 \%$ (words) and $72.3 \%$ (isolated letters).

Turning now to the data for exposure durations, a main effect was found for mask type: stimuli were presented in backward-masked displays for an average of $13.37 \mathrm{msec}$ (12.21 msec for words, $14.52 \mathrm{msec}$ for isolated letters), while the same stimuli were presented in forward-masked displays for an average of $47.60 \mathrm{msec}$ [46.53 msec for words, $48.66 \mathrm{msec}$ for isolated letters; $\left.F(1,14)=258.12, M S_{\mathrm{e}}=1.65, p<.0001\right]$. A main effect was also found for stimulus type: mean exposure durations were $2.22 \mathrm{msec}$ longer $(S E= \pm 0.25$ ) for isolated letters than they were for words $\left[F(1,14)=16.47, M S_{\mathrm{e}}=\right.$ $0.52, p<.001]$. However, this effect showed no sign of interacting with mask type $\left[F(1,14)=0.28, M S_{\mathrm{e}}=\right.$
$1.64, p=.60$ ]; indeed, the word advantage was removed to the same extent in each mask condition (see accuracy data above) by virtually identical increases in exposure duration for isolated letters [2.13 msec $(S E= \pm 0.85)$ for forward-masked displays and $2.31 \mathrm{msec}(S E= \pm 0.28)$ for backward-masked displays]. Moreover, the negligible difference $(0.18 \mathrm{msec})$ between the two increases was in the opposite direction to that predicted by the hypothesis that the longer exposure durations observed with forward pattern masks in Experiment 2 concealed a greater delay between the perception of letters in words and isolated letters relative to that produced by backward pattern masks. Consequently, while the findings of Experiment 2 show that stimulus perception in general occurred over a longer time scale with forward pattern masks, the findings of Experiment 3 indicate that this difference does not extend to the temporal relationship between word and letter perception in each mask condition because forward-and backward-masked forms of the WLP were both removed by essentially the same increase in exposure duration.

\section{Discussion}

In the light of these findings, it seems likely that the overall increase in masking observed with forwardmasked displays in Experiments 2 and 3 was due to increased interference at a peripheral level of analysis, far removed from representations for words and letters. Consequently, while obtaining an advantage for words over isolated letters with forward pattern masks provides problems for contemporary accounts of the WLP, the longer overall exposure durations observed with forward-masked displays in Experiments 2 and 3 and the absence of a concomitant influence on the relative perceptibility of words and isolated letters are both consistent with contemporary theories concerning the different loci of masking effects. We shall return to this point in the General Discussion section.

At first sight, the shift in exposure duration for isolated letters (a little over $2 \mathrm{msec}$ ) required to remove each form of the WLP observed in Experiment 2 may appear to be implausibly small. However, the finding that such a small duration shift can remove a substantial word advantage does have a precedence, at least for backward-masked displays. Taylor and Chabot (1978) examined the influence of exposure duration on the relative perceptibility of words and isolated letters in backward pattern-masked displays. Stimuli were presented over a range of exposure durations, and their relative perceptibility was determined by the interval between target offset and mask onset that was necessary to achieve a particular performance criterion (accurately reporting four consecutive targets). When the duration of isolated letters was increased by just $2 \mathrm{msec}$ (e.g., from $8 \mathrm{msec}$ to $10 \mathrm{msec}$ ), the perceptual advantage observed for words when both words and isolated letters were presented at the same exposure duration (for this example, $8 \mathrm{msec}$ ) was completely removed. It should be said that some interobserver variation in performance was apparent in Taylor and Chabot's experiment (only two subjects were used) and that no data were obtained for 
forward-masked displays. Nevertheless, in terms of the effects of exposure duration on word superiority over isolated letters, it appears that a shift of $2 \mathrm{msec}$ may indeed be sufficient to remove an advantage for words.

Finally, despite selectively reducing the exposure durations for words in Experiment 3, an identical negligible word advantage $(0.9 \%)$ remained in each mask condition. Thus, both forms of the WLP were equally resistant to attempts at removal and, while forward and backward pattern masks produced very similar effects on the relative accuracy with which letters in words and isolated letters were reported, the absolute shift in exposure duration required to remove these word advantages completely is likely to be fractionally greater than the mean of 2.2 msec obtained in Experiment 3.

\section{GENERAL DISCUSSION}

The goal of the experiments reported in this article was to examine the role of pattern masks in the WLP. More specifically, contemporary interpretations of previous findings in this area propose that the WLP observed under abbreviated viewing conditions critically depends on the use of backward pattern masks. This, in turn, has inspired models of word and letter perception (e.g., the IAM and the HM) to account for the WLP in terms of the ability of pattern masks to disrupt ongoing processes of word and letter analysis and to overlook the possibility that the WLP may be produced under forward-masked conditions. Contrary to these accounts, however, the experiments reported in this article show that the WLP can be produced when stimuli are preceded by a pattern mask and followed by a completely blank field.

One immediate implication of the word advantage observed with forward pattern masks is that it cannot be accommodated by existing explanations of the WLP observed with backward pattern masks. The IAM and HM propose that the role of pattern masking in the WLP is explained by the behavior of a system in which stimulus processing gets under way before masking disruption takes place. However, under forward-masked conditions, stimulus presentation will impinge on a system already affected by mask activations. Thus, while accurate stimulus recognition under backward-masked conditions can be characterized as the resistance of ongoing stimulus processing to subsequent masking effects, accurate stimulus recognition under forward-masked conditions can be characterized as the overcoming of ongoing masking effects by subsequent stimulus processing.

The implications of the differences between these two processing scenarios become clearer when attempts are made to simulate the WLP observed with forward-masked displays using the software implementation of the IAM provided by McClelland and Rumelhart (1988). ${ }^{4}$ This software simulates the hierarchical IAM arrangement of units at each of three processing levels: feature, letter, and word. At the feature level, there is a set of units for features in each of four letter positions. Within each set, units respond to the presence of each of the line segments composing the simple font adopted by Rumelhart and $\mathrm{Si}$ ple (1974). At the letter level, there are four sets of letter units (each set contains units for each of the 26 letters in the English alphabet), one set for each position in a four-letter word. At the word level, there is a single set of detectors for each of 1,179 four-letter words.

The software receives simulated visual input from a keyboard or text file (i.e., the simulation does not have eyes) and so describes only the central aspects of the perceptual processes involved. Notwithstanding this restriction, the model can be used to simulate trials from psychological experiments. For example, several successive displays within a single "experimental trial" may be specified, with each display characterized by an onset time and an array of "visual" information. In this way, trials can be made up of displays of any sequence of words, isolated letters, and pattern masks (the latter composed of individual characters simulating an overstruck $\mathrm{X}-\mathrm{O}$ combination), although the software normalizes each input into a consistent font by redefining each letter and mask character in terms of the presence or absence of 14 letter features (after Rumelhart \& Siple, 1974).

The simulation operates in discrete time slices (or ticks), updating the activations of all of the units in the system once in each cycle, and assumes that the information used to respond in backward-masked displays is that present in the system just before the onset of the poststimulus mask (McClelland \& Rumelhart, 1988). However, McClelland and Rumelhart (1988, p. 214) assert that when stimuli are not followed by a pattern mask, the point in processing at which a response is chosen occurs "after activation and response strengths reach their asymptotic values." The model's performance under forward-masked conditions was examined using this assumption.

The basic parameters of the simulation, such as decay rate, maximum activation, and minimum activation, were not altered and words and isolated letters were processed for as long as it took the activations produced by these stimuli to reach asymptote. A forward mask was "presented" to the model for 16 cycles, and was followed by a word or isolated letter until activations reached asymptote. The outcome of this simulation revealed that, at asymptote, words and isolated letters had a similar correct response probability ( .80 for words, .83 for isolated letters; see Figure 4) and so simulate the findings of previous research from which the model was derived, namely, that the WLP disappears when no backward pattern mask is used. In this form, however, the simulation clearly fails to accommodate the forward-masked WLP reported in this article.

However, while McClelland and Rumelhart's (1988) assumption that readout takes place at asymptote when no backward pattern mask is presented may be appropriate for displays in which no pattern mask at all (post- or prestimulus) is used, it may be unreasonable to make the same assumption about readout under forward-masked conditions. For example, under forward-masked condi- 


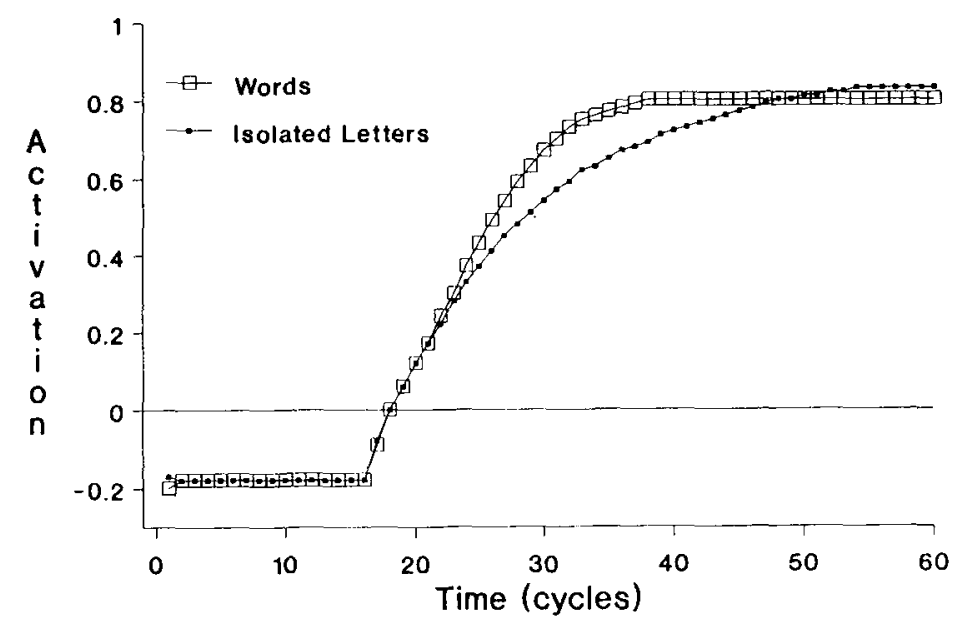

Figure 4. The time course of activations produced by the IAM software (McClelland \& Rumelhart, 1988) for words and isolated letters under forward-masked conditions. Masks were replaced by targets after 16 cycles. Note the advantage for words which begins to appear at 22 cycles (i.e., 6 cycles after target onset) and which peaks at 32 cycles (i.e., 16 cycles after target onset).

tions, performance may be limited by the efficiency with which the identity of a target can be extracted from a rapidly fading composite image of target and mask. In other words, a severe temporal limitation may be imposed on the analysis of target information in forward-masked displays even though target processing is not interrupted by mask activation. This, in turn, may mean that the probability of responding correctly will be higher before asymptote is reached (cf. the IAM) and, therefore, that subjects may adopt this strategy in forward-masked experiments. The simulation of forward masking provided by the IAM software does not reflect this sequence of activation. However, an inspection of the levels of activation produced by words and isolated letters in Figure 4 reveals that, prior to the asymptotic levels produced by the simulation, an advantage for words does exist. Specifically, after target information has been processed for 6 cycles, a gap begins to form between the levels of activation for words and isolated letters that peaks at $16 \mathrm{cy}$ cles to form a substantial word advantage (a difference in activation of .14). Therefore, if we replace the assumption (made by McClelland \& Rumelhart, 1988) that when no backward pattern mask is presented, responses will be read out when activation reaches asymptote with the assumption that forward pattern-masked stimuli have only a finite time in which to be processed (in our simulation, 16 cycles), the IAM is able to simulate the forwardmasked form of the WLP obtained with human subjects. Indeed, while the absolute levels of performance for words and letters in the simulation were slightly different from those obtained in the experiments reported here, a similarsized WLP was obtained with backward-and forwardmasked simulations (.13 for backward masks and .14 for forward masks; see Figure 5), which concurs with the findings of Experiments 2 and 3. Thus, despite the spe- cial role that the IAM assigns to backward pattern masks, the model has some success in accounting for the forwardmasked form of the WLP reported in this article.

It is worth noting at this point that when a WLP was observed with human subjects under forward-masked conditions, target exposure durations were considerably longer than they were under backward-masked conditions (Experiments 2 and 3). Thus, it may seem strange that in the forward- and backward-masked simulations provided by the IAM, a similar-sized WLP was produced in each masking condition after target stimuli had been processed for a similar number of cycles (for forward masks, 16 cycles; for backward masks, 14 cycles). However, it remains to be seen exactly how exposure duration relates to processing time. For example, one explanation of the longer exposure durations observed with forward-masked displays in Experiments 2 and 3 is that this difference reflects increased disruption at peripheral levels of processing and that the dynamics of processing within the word-recognition module are similarly disrupted by forward and backward masks (see Discussion section following Experiment 3); indeed, this explanation is supported by the findings of Experiment 3 . Thus, the apparent temporal discrepancy between the IAM's simulation and the human data is not fatal.

But precisely why should similar forms of the WLP be produced with forward- and backward-masked displays? Following the arguments of Jordan and de Bruijn (1993), one possibility is that the identity of a target can be extracted from misleading mask information more efficiently for words than it can for isolated letters and that this situation occurs equally for both backward and forward pattern-masked displays. Specifically, in view of the diminutive size of isolated letters, integration between target and mask would place isolated letters in a dispropor- 

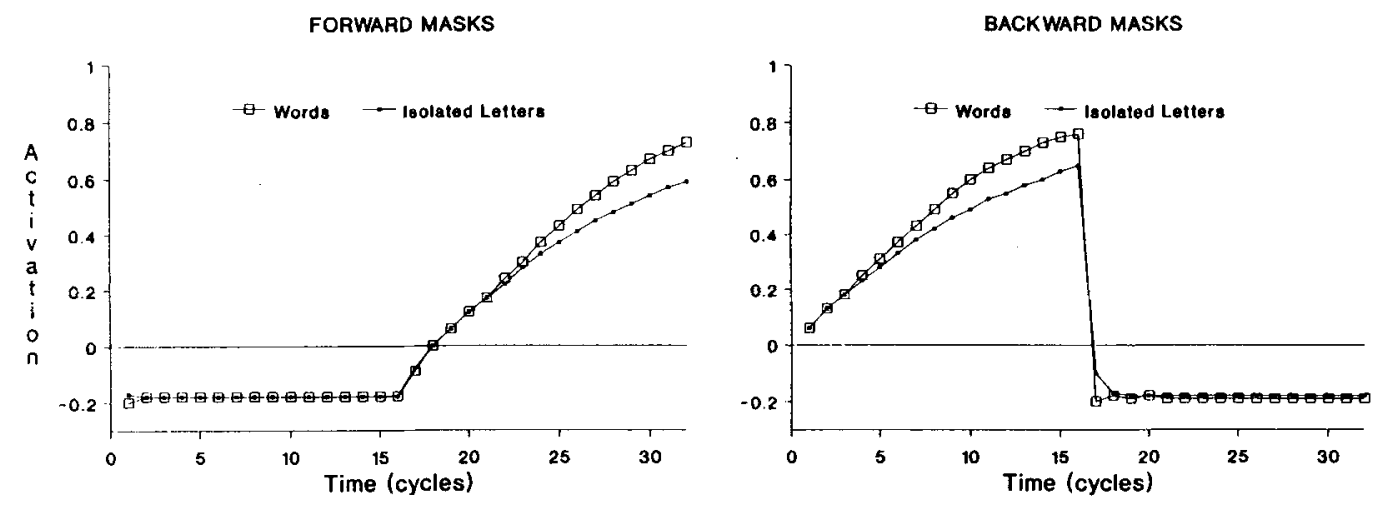

Figure 5. A comparison of the levels of activation produced by the IAM software (McClelland \& Rumelhart, 1988) under forward- and backward-masked conditions for words and isolated letters. In each mask condition, activation levels are shown for 16 cycles of mask presentation and 16 cycles of target presentation. For forward masks, the word advantage peaked (.14) at 32 cycles (i.e., 16 cycles after target onset); for backward masks, the word advantage peaked (.13) at 14 cycles (i.e., 14 cycles after target onset).

tionately large background of mask contours. Consequently, the two forms of the WLP observed with backward and forward pattern masks may reflect differences in the discriminability of words and isolated letters from the background provided by their respective masks, such that a word advantage is inspired because differences in relative mask size mean that the location and extent of each word are more easily discriminated than are the location and extent of each isolated letter in a composite percept of target and mask.

Support for the view that the WLP may be inspired by the ease with which targets are discriminated from pattern masks comes from Prinzmetal's (1992) study in which a word advantage over isolated letters was found when targets were physically embedded in a pattern-mask background. Given that the present article is the first to report the forward-masked form of the WLP, the role of target-mask discriminability in this form of the phenomenon remains to be examined; however, Jordan and de Bruijn (1993) provide evidence of the influence of targetmask discriminability on the backward-masked form of the WLP. As Jordan and de Bruijn point out, a number of studies have shown that targets are difficult to discriminate when the background in which the target is embedded contains features that are also present in the target item (e.g., Beck, 1966, 1967; Beck \& Ambler 1972, 1973; Treisman \& Gormican, 1988), and that the time taken to discriminate a target from its background increases with the size of the background array, particularly when targets contain no one feature that distinguishes them from their background (e.g., Duncan, 1979, 1987; Duncan \& Humphreys, 1989; Humphreys, Quinlan, \& Riddoch, 1989; Treisman \& Gelade, 1980). Thus, Jordan and de Bruijn reasoned that if the WLP reflects the operation of similar discrimination processes involving targets and masks, the WLP itself should be sensitive to the size of the masking stimulus. Over a series of experiments, Jor- dan and de Bruijn examined the effects of varying the size of each pattern mask relative to that of the target being masked. They found that the WLP was produced, removed, and even reversed simply by altering the width of each mask relative to the width of the target it was masking even though, at all times, masks covered targets. For example, when pattern masks matched the width of words and exceeded the width of isolated letters, isolated letters were presented in a disproportionately large masking field and the WLP was observed. However, when the width of each mask matched the width of each target (word or isolated letter) or when masks exceeded the width of words and isolated letters by the same proportional amount, the WLP was reversed (i.e., performance was better for isolated letters than it was for words).

It may be premature to dismiss the idea that mask interruption contributes to the WLP observed with backwardmasked displays. Nevertheless, one parsimonious interpretation of similar effects produced by forward and backward masks on the relative perceptibility of words and isolated letters reported in this article is that interruption plays no part in either form of the WLP. Indeed, the similar effects observed with forward and backward masks have widespread implications for contemporary views on masking. In particular, many researchers argue that forward masks exert most of their influence at peripheral stages of processing, while only backward masks exert effects on central (cortical) stages of target perception (e.g., Greenspon \& Eriksen, 1968; Kietzman et al., 1971; Michaels \& Turvey, 1979; Moscovitch, 1986; Schiller, 1966; Schiller \& Smith, 1965; Smith \& Schiller, 1966; Sperling, 1960, 1965; Turvey, 1973; see Discussion section, Experiment 2). For example, when discussing the effects of forward and backward pattern masks, Turvey (1973, p. 34) concludes that "forward and backward masking can both occur peripherally, but only backward masking occurs to any degree centrally." Moreover, it 
is apparent from the accounts of the IAM and HM, where the WLP is produced by selective disruption of representations for letters and words, that both models assume that backward pattern masks exert their critical influence on the relative perceptibility of words and isolated letters at a central (cortical) stage of processing (presumably, for most individuals, in the left hemisphere; e.g., Geschwind, 1991, although see Bishop, 1988). Nevertheless, the effects observed for forward and backward pattern masks in the experiments reported in this article suggest either that the critical role of pattern masks in the WLP occurs at a peripheral stage (where both forward and backward pattern masks may be exerting an effect) or that forward pattern masks have more of an influence on central stages of target perception than previously thought. Further comparisons of the effects of backward and forward pattern masks on the relative perceptibility of words and letters, using monoptic and dichoptic presentations of targets and masks, are required to help resolve this issue.

In conclusion, while contemporary accounts of the WLP propose a critical role for backward pattern masks, a similar WLP is produced when targets are preceded by a pattern mask and followed by a blank field. Moreover, the similar effects produced by forward and backward pattern masks on the relative perceptibility of isolated letters and letters in words suggest that the role of pattern masks in both forms of the WLP may be best explained by considering aspects of masking common to both types of mask; target-mask discrimination provides one such explanation.

\section{REFERENCES}

BECK, J. (1966). Effect of orientation and of shape similarity on perceptual grouping. Perception \& Psychophysics, 1, 300-302.

BECK, J. (1967). Perceptual grouping produced by line figures. Perception \& Psychophysics, 2, 491-495.

BECK, J., \& AMBLER, B. (1972). Discriminability of differences in line slope and in line arrangement as a function of mask delay. Perception \& Psychophysics, 12, 33-38.

BECK, J. \& AMBLER, B. (1973). The effects of concentrated and distributed attention on peripheral acuity. Perception \& Psychophysics, 14, 225-230.

Bishop, D. V. M. (1988). Can the right hemisphere mediate language as well as the left? A critical review of recent research. Cognitive Neuropsychology, 5, 353-367.

BREITMEYER, B. G. (1984). Visual masking: An integrative approach. Oxford: Oxford University Press, Clarendon Press.

BReITMEYER, B. G., \& GANZ, L. (1976). Implications of sustained and transient channels for theories of visual pattern masking, saccadic suppression, and information processing. Psychological Review, 83, 1-36.

Carr, T. H., Davidson, B. J., \& Hawkins, H. L. (1978). Perceptual flexibility in word recognition: Strategies affect orthographic computation but not lexical access. Journal of Experimental Psychology: Human Perception \& Performance, 4, 674-690.

Carr, T. H., Lehmkuhle, S. W., Kottas, B., Astor-Stetson, E. C., \& ARNOLD, D. (1976). Target position and practice in the identification of letters in varying contexts: A word superiority effect. Perception \& Psychophysics, 19, 412-416.

Carroll, J. B., Davies, P., \& Richman, B. (1971). The American Heritage word frequency book. Boston: Houghton-Mifflin.

Duncan, J. (1979). Divided attention: The whole is more than the sum of its parts. Journal of Experimental Psychology: Human Perception \& Performance, 5, 216-228.

DUNCAN, J. (1987). Attention and reading: Wholes and parts in shape recognition. In M. Coltheart (Ed.), Attention and performance XII (pp. 39-61). Hove, U.K.: Erlbaum.

DUNCAN, J., \& Humphreys, G. W. (1989). Visual search and stimulus similarity. Psychological Review, 96, 433-458.

ERIKSEN, C. W. (1980). The use of a visual mask may seriously confound your experiment. Perception \& Psychophysics, 28, 89-92.

EsTEs, W. K. (1975a). The locus of inferential and perceptual processes in letter identification. Journal of Experimental Psychology: General, 104, 122-145

ESTES, W. K. (1975b). Memory, perception, and decision in letter identification. In R. Solso (Ed.), Information processing and cognition: The Loyola Symposium (pp. 3-30). Potomac, MD: Erlbaum.

Felsten, G., \& Wasserman, G. S. (1980). Visual masking: Mechanisms and theories. Psychological Bulletin, 88, 329-353.

GANZ, L. (1975). Temporal factors in visual perception. In E. C. Carterette \& M. P. Friedman (Eds.), Handbook of perception (Vol. 5, pp. 169-231). New York: Academic Press.

GESCHWIND, N. (1991). Specializations of the human brain. In W. S.-Y. Wang (Ed.), The emergence of language: Development and evolution (pp. 72-87). New York: W. H. Freeman.

GOLDEN, R. M. (1986). A developmental neural model of visual word perception. Cognitive Science, 10, 241-276.

Greenspon, T. S., \& ERIKSEN, C. W. (1968). Interocular nonindependence. Perception \& Psychophysics, 3, 93-96.

hawkins, H. L., Reicher, G. M., Rogers, M., \& Peterson, L. (1976). Flexible coding in word recognition. Journal of Experimental Psychology: Human Perception \& Performance, 2, 380-385.

HoleNDER, D. (1979). Identification of letters in words and of single letters with pre- and postknowledge vs. postknowledge of the alternatives. Perception \& Psychophysics, 25, 313-318.

HumphreYs, G. W., Quinlan, P. T., \& Rindoch, M. J. (1989). Grouping processes in visual search: Effects with single- and combinedfeature targets. Journal of Experimental Psychology: General, 118, 258-279.

Johnston, J. C. (1978). A test of the sophisticated guessing theory of word perception. Cognitive Psychology, 10, 123-153.

JoHNSTON, J. C. (1981). Understanding word perception: Clues from studying the word superiority effect. In O. Tzeng \& H. Singer (Eds.), Perception of print: Reading research in experimental psychology (pp. 65-84). Hillsdale, NJ: Erlbaum.

Johnston, J. C., \& MCCLElland, J. L. (1973). Visual factors in word perception. Perception \& Psychophysics, 14, 365-370.

Johnston, J. C., \& MCClelland, J. L. (1974). Perception of letters in words: Seek not and ye shall find. Science, 184, 1192-1194.

Johnston, J. C., \& MCClelland, J. L. (1980). Experimental tests of a hierarchical model of word recognition. Journal of Verbal Learning \& Verbal Behavior, 19, 503-524.

JoRDAN, T. R. (1990). Presenting words without interior letters: Superiority over single letters and influence of postmask boundaries. Journal of Experimental Psychology: Human Perception \& Performance, 16, 893-909.

JoRDAN, T. R. (in press). Contrasting effects of letter fragment masks and non-letter fragment fields on alphabetic targets: Examining the role of mask configuration. Journal of Experimental Psychology: Human Perception \& Performance.

JORDAN, T. R., \& DE BRUIJN, O. (1993). Word superiority over isolated letters: The neglected role of flanking mask contours. Journal of Experimental Psychology: Human Perception \& Performance, 19, 549-563.

JoRDAN, T. R., \& MARTIN, C. D. (1987). The importance of visual angle in word recognition: A "shrinking screen" modification for visual displays. Behavior Research Methods, Instruments, \& Computers, 19, 307-310.

Juola, J. F., LeavitT, D. D., \& Choe, C. S. (1974). Letter identification in word, nonword, and single-letter displays. Bulletin of the Psychonomic Society, 4, 278-280.

KaHNEMAN, D. (1968). Method, findings, and theory in studies of visual masking. Psychological Bulletin, 70, 404-426.

Kietzman, M. L., Boyle, R. C., \& Lindsley, D. B. (1971), Perceptual masking: Peripheral vs central factors. Perception \& Psychophysics, 9(4), 350-352.

KOLERS, P. A. (1968). Some psychological aspects of pattern recogni- 
tion. In P. A. Kolers \& M. Eden (Eds.), Recognizing patterns. Boston: MIT Press.

Marchetti, F. M., \& Mewhort, D. J. K. (1986). On the wordsuperiority effect. Psychological Research, 48, 23-35.

Massaro, D. W., \& Klitzke, D. (1979). The role of lateral masking and orthographic structure in letter and word recognition. Acta Psychologica, 43, 413-426.

MCClelland, J. L. (1985). Putting knowledge in its place: A scheme for programming parallel structures on the fly. Cognitive Science, 9 , 113-146.

MCCleLLAND, J. L. (1986). The programmable blackboard model of reading. In J. L. McClelland \& D. E. Rumelhart (Eds.), Parallel distributed processing: Explorations in the microstructure of cognition (Vol. 2, pp. 122-169). Cambridge, MA: MIT Press.

MCClelland, J. L. (1991). Stochastic interactive processes and the effect of context on perception. Cognitive Psychology, 23, 1-44.

MCClelland, J. L., \& Rumelhart, D. E. (1981). An interactive activation model of context effects in letter perception: Part 1. An account of basic findings. Psychological Review, 88, 375-407.

McClelland, J. L., \& Rumelhart, D. E. (1988). Explorations in parallel distributed processing. Cambridge, MA: MIT Press.

Mewhort, D. J. K., \& Johns, E. E. (1988). Some tests of the interactive-activation model for word identification. Psychological Research, 50, 135-147.

Michaels, C. F., \& Turvey, M. T. (1979). Central sources of visual masking: Indexing structures supporting seeing at a single, brief glance. Psychological Research, 41, 1-61.

Moscovitch, M. (1986). Hemispheric specialization, interhemispheric codes, and transmission times: Inferences from visual masking studies in normal people. In F. Lepore, M. Ptito, \& H. H. Jasper (Eds.), Two hemispheres-one brain: Functions of the corpus callosum (pp. 483-510). New York: Liss.

PaAP, K. R., Newsome, S. L., McDonald, J. E., \& SchVaneveldT, R. W. (1982). An activation-verification model for letter and word recognition: The word-superiority effect. Psychological Review, 89, 573-594.

Prinzmetal, W. (1992). The word-superiority effect does not require a T-scope. Perception \& Psychophysics, 51, 473-484.

Purcell, D. G., \& Stanovich, K. E. (1982). Some boundary conditions for a word superiority effect. Quarterly Journal of Experimental Psychology, 34A, 117-134.

ReICHER, G. M. (1969). Perceptual recognition as a function of meaningfulness of stimulus material. Journal of Experimental Psychology, $\mathbf{8 1}$, 275-280.

Rumelhart, D. E., \& MCClelland, J. L. (1982). An interactive activation model of context effects in letter perception: Part 2 . The contextual enhancement effect and some tests and extensions of the model. Psychological Review, 89, 60-94.

Rumelhart, D. E., \& Siple, P. (1974). The process of recognizing tachistoscopically presented words. Psychological Review, 81, 99-118.
SCHILLER, P. H. (1966). Forward and backward masking as a function of relative overlap and intensity of test and masking stimuli. Perception \& Psychophysics, 1, 161-164.

SCHILlER, P. H., \& SMITH, M. C. (1965). A comparison of forward and backward masking. Psychonomic Science, 3, 77-78.

Smith, M. C., \& Schiller, P. H. (1966). Forward and backward masking: A comparison. Canadian Journal of Psychology, 20, 337.342.

SpERLING, G. (1960). The information available in brief visual presentations. Psychological Monographs, 74 (Whole No. 498).

SPERLING, G. (1965). Temporal and spatial visual masking: I. Masking by impulse flash. Journal of the Optical Society of America, $\mathbf{5 5}$, 541-559.

TAYloR, G. A., \& Сhaвot, R. J. (1978). Differential backward masking of words and letters by masks of varying orthographic structure. Memory \& Cognition, 6, 629-635.

Treisman, A., \& Gelade, G. (1980). A feature integration theory of attention. Cognitive Psychology, 12, 97-136.

Treisman, A., \& Gormican, S. (1988). Feature analysis in early vision: Evidence from search asymmetries. Psychological Review, 95 , $15-48$.

Turver, M. T. (1973). On peripheral and central processes in vision. Psychological Review, 80, 1-52.

Wheeler, D. D. (1970). Processes in word recognition. Cognitive Psychology, 1, 59-85.

\section{NOTES}

1. It is interesting to note that in the seminal comparison of the effects of pattern masks and blank fields on the WLP reported by Johnston and McClelland (1973), each pattern-masked target was followed and preceded by a pattern mask. In hindsight, it is curious that the findings of this study led to so much importance being attached to the use of backward pattern masks in the WLP.

2. It should be emphasized that we are not disputing that the overall time course of target processing is longer in forward-masked displays, but are debating whether the time course separating the perception of words and isolated letters (which, presumably, inspires the WLP) in each mask condition is longer in forward-masked displays.

3. Reaction-time studies, in which the dependent variable was the time taken to identify the critical letters in each condition, were considered. However, a pilot study indicated that the timing of overt responses was not a sufficiently sensitive technique to reveal temporal differences between the perceptual processing of words and isolated letters under abbreviated viewing conditions. Consequently, the reaction-time paradigm was rejected.

4. The HM was not available to us in software form and so could not be tested in this way.

(Manuscript received January 26, 1993; revision accepted for publication June 30, 1993.) 